\title{
The order of principal congruences of a bounded lattice
}

\author{
G. GRÄTZER
}

\begin{abstract}
We characterize the order of principal congruences of a bounded lattice as a bounded ordered set. We also state a number of open problems in this new field.
\end{abstract}

\section{Introduction}

1.1. Congruence lattices. Let $A$ be a lattice (resp., join-semilattice with zero). We call $A$ representable if there exist a lattice $L$ such that $A$ is isomorphic to the congruence lattice of $L$, in formula, $A \cong \operatorname{Con} L$ (resp., $A$ is isomorphic to the join-semilattice with zero of compact congruences of $L$, in formula, $\left.A \cong \operatorname{Con}_{\mathrm{c}} L\right)$.

For over 60 years, one of lattice theory's most central conjectures was the following:

Characterize representable lattices as distributive algebraic lattices.

(Or equivalently: Characterize representable join-semilattices as distributive join-semilattice with zero.) This conjecture was refuted in F. Wehrung [15.

The finite case of this field is surveyed in my book [2. The infinite casealong with some research fields connected with it - is surveyed in four chapters in [12], three by F. Wehrung and one by me.

1.2. Principal congruences. In this note, we deal with Princ $L$, the order of principal congruences of a lattice $L$. Observe that

(a) Princ $L$ is a directed order with zero.

(b) $\mathrm{Con}_{\mathrm{c}} L$ is the set of compact elements of Con $L$, a lattice theoretic characterization of this subset.

(c) Princ $L$ is a directed subset of $\operatorname{Con}_{\mathrm{c}} L$ containing the zero and join-generating $\operatorname{Con}_{\mathrm{c}} L$; there is no lattice theoretic characterization of this subset.

Figure 1 shows the lattice $\mathrm{N}_{7}$ and its congruence lattice $\mathrm{B}_{2}+1$. Note that Princ $\mathrm{N}_{7}=$ Con $\mathrm{N}_{7}-\{\gamma\}$. While in the standard representation $K$ of $\mathrm{B}_{2}+1$ as a congruence lattice (G. Grätzer and E. T. Schmidt [6]; see also in my books [2], [3]), we have Princ $K=\operatorname{Con} K$. This example shows that Princ $L$ has no lattice theoretic description in Con $L$.

Presented by ...

Received ...; accepted in final form ...

2010 Mathematics Subject Classification: Primary: 06B10; Secondary: 06A06.

Key words and phrases: principal congruence, order. 


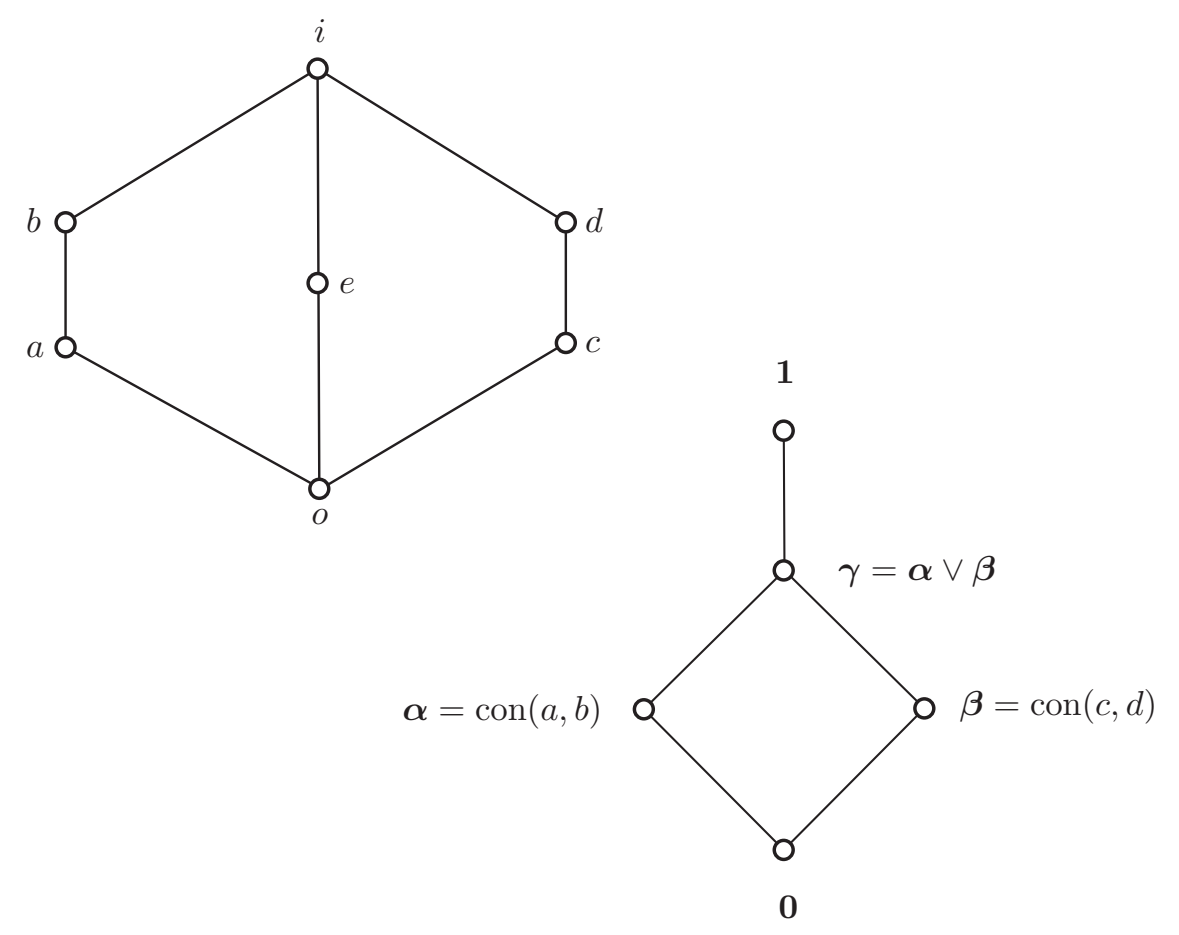

FIgURE 1. The lattice $\mathrm{N}_{7}$ and its congruence lattice

It was pointed out in G. Grätzer and E. T. Schmidt [5] that for every algebra $\mathfrak{A}$ we can construct an algebra $\mathfrak{B}$ such that $\operatorname{Con} \mathfrak{A} \cong \operatorname{Con} \mathfrak{B}$ and Princ $\mathfrak{B}=$ $\mathrm{Con}_{\mathrm{c}} \mathfrak{B}$.

For a long time, we have tried to prove such a result for lattices but we have been unable to construct even a proper congruence-preserving extension for a general lattice; see the discussion in G. Grätzer and E. T. Schmidt 7 . This logjam was broken in G. Grätzer and F. Wehrung [11] by introducing the boolean triple construction. G. Grätzer and E. T. Schmidt 9] uses this construction to prove the following result:

Theorem 1. Every lattice $L$ has a congruence-preserving extension $K$ satisfying

$$
\text { Princ } K=\operatorname{Con}_{\mathrm{c}} K \text {. }
$$

So if a distributive join-semilattice with zero $S$ can be represented as $\operatorname{Con}_{\mathrm{c}} L$ for a lattice $L$, then $S$ can also be represented as Princ $K$ for a lattice $K$. This is a further illustration of the fact that Princ $L$ has no lattice theoretic description in Con $L$.

1.3. The result. For a bounded lattice $L$, the order Princ $K$ is bounded. We now state the converse. 
Theorem 2. Let $P$ be an order with zero and unit. Then there is a bounded lattice $K$ such that

$$
P \cong \operatorname{Princ} K .
$$

If $P$ is finite, we can construct $K$ as a finite lattice.

We construct $K$ as a lattice of length 5 . So $K$ is complete. All of its congruences are complete. So we also get Theorem 2 for principal congruences of complete lattices and for principal complete congruences of complete lattices.

1.4. Problems. The real purpose of this note is to state some of the many open problems in this field.

\subsubsection{General lattices.}

Problem 1. Can we characterize the order Princ $L$ for a lattice $L$ as a directed order with zero?

Even more interesting would be to charaterize the pair $P=\operatorname{Princ} L$ in $S=\operatorname{Con}_{\mathrm{c}} L$ by the properties that $P$ is a directed order with zero that joingenerates $S$. We have to rephrase this so it does not require a solution of the congruence lattice characterization problem.

Problem 2. Let $S$ be a representable join-semilattice. Let $P \subseteq S$ be a directed order with zero and let $P$ join-generate $S$. Under what conditions is there a lattice $K$ such that $\operatorname{Con}_{\mathrm{c}} K$ is isomorphic to $S$ and under this isomorphism Princ $K$ corresponds to $P$ ?

For a lattice $L$, let us define a valuation $v$ on $\operatorname{Con}_{\mathrm{c}} L$ as follows: for a compact congruence $\boldsymbol{\alpha}$ of $L$, let $v(\boldsymbol{\alpha})$ be the smallest integer $n$ such that the congruence $\boldsymbol{\alpha}$ is the join of $n$ principal congruences. A valuation $v$ has some obvious properties, for instance, $v(\mathbf{0})=0$ and $v(\boldsymbol{\alpha} \vee \boldsymbol{\beta}) \leq v(\boldsymbol{\alpha})+v(\boldsymbol{\beta})$. Note the connection with Princ $L$ :

$$
\text { Princ } L=\left\{\boldsymbol{\alpha} \in \operatorname{Con}_{\mathrm{c}} L \mid v(\boldsymbol{\alpha}) \leq 1\right\} .
$$

Problem 3. Let $S$ be a representable join-semilattice. Let $v$ map $S$ to the natural numbers. Under what conditions is there an isomorphism $\varphi$ of $S$ with $\mathrm{Con}_{\mathrm{c}} K$ for some lattice $K$ so that under $\varphi$ the map $v$ corresponds to the valuation on $\mathrm{Con}_{\mathrm{c}} K$ ?

1.4.2. Finite lattices. Let $D$ be a finite distributive lattice. In G. Grätzer and E. T. Schmidt [6], we represent $D$ as the congruence lattice of a finite lattice $K$ in which all congruences are principal (that is, $\operatorname{Con} K=\operatorname{Princ} K$ ).

Problem 4. Let $D$ be a finite distributive lattice. Let $Q$ be a subset of $D$ satisfying $\{0,1\} \cup$ Ji $K \subseteq Q \subseteq D$. When is there a finite lattice $K$ such that Con $K$ is isomorphic to $D$ and under this isomorphism Princ $K$ corresponds to $Q$ ?

In the finite variant of Problem 3 , we need an additional property. 
Problem 5. Let $S$ be a finite distributive lattice. Let $v$ be a map of $D$ to the natural numbers satisfying $v(0)=0, v(1)=1$, and $v(a \vee b) \leq v(a)+v(b)$ for $a, b \in D$. When is there an isomorphism $\varphi$ of $D$ with $\operatorname{Con} K$ for some finite lattice $K$ such that under $\varphi$ the map $v$ corresponds to the valuation on Con $K$ ?

1.4.3. Special classes of lattices. There are many problems that deal with lattices with we only mention two.

In G. Grätzer, H. Lakser and E. T. Schmidt [4] and G. Grätzer and E. T. Schmidt [8], we investigate congruence lattices of finite semimodular lattices.

Problem 6. In Theorem 2, can we construct a semimodular lattice?

Problem 7. In Problems 2 and 3 in the finite case, can we construct a finite semimodular lattice $K$ ?

In E. T. Schmidt 14] (see also G. Grätzer and E. T. Schmidt [10), for a finite distributive lattice $D$, a countable modular lattice $M$ is constructed with Con $M \cong D$.

Problem 8. In Theorem 2, for a finite $P$, can we construct a countable modular lattice $K$ ?

For the background for some other classes of lattices, see my book 2 .

1.4.4. Complete lattices. The techniques developed in this note may be applicable to solve the following problem:

Problem 9. Let $K$ be a bounded lattice. Does there exist a complete lattice $L$ such that Con $K \cong \operatorname{Con} L$ ?

1.4.5. Algebras in general. Some of these problems seem to be of interest for algebras other than lattices as well.

Problem 10. Can we characterize the order Princ $\mathfrak{A}$ for an algebra $\mathfrak{A}$ as an order with zero?

Problem 11. For an algebra $\mathfrak{A}$, how is the assumption that the unit congruence $\mathbf{1}$ is compact reflected in the order Princ $\mathfrak{A}$ ?

Problem 12. Let $\mathfrak{A}$ be an algebra and let Princ $\mathfrak{A} \subseteq Q \subseteq \operatorname{Con}_{\mathrm{c}} \mathfrak{A}$. Does there exist an algebra $\mathfrak{B}$ such that $\operatorname{Con} \mathfrak{A} \cong \operatorname{Con} \mathfrak{B}$ and under this isomorphism $Q$ corresponds to Princ $\mathfrak{B}$ ?

Problem 13. Extend the concept of valuation to algebras. State and solve Problem 3 for algebras.

Problem 14. Can we sharpen the result of G. Grätzer and E. T. Schmidt [5]: every algebra $\mathfrak{A}$ has a congruence-preserving extension $\mathfrak{B}$ such that Con $\mathfrak{A} \cong$ Con $\mathfrak{B}$ and Princ $\mathfrak{B}=\operatorname{Con}_{\mathrm{c}} \mathfrak{B}$.

I do not even know whether every algebra $\mathfrak{A}$ has a proper congruencepreserving extension $\mathfrak{B}$. 


\section{The construction}

For a bounded order $Q$, let $Q^{-}$denote the order $Q$ with the bounds removed. Let $P$ be the order in Theorem 2. Let 0 and 1 denote the zero and unit of $P$, respectively. We denote by $P^{\mathrm{d}}$ those elements of $P^{-}$that are not comparable to any other element of $P^{-}$, that is,

$$
P^{\mathrm{d}}=\left\{x \in P^{-} \mid x \| y \text { for all } y \in P^{-}, y \neq x\right\} .
$$

2.1. The lattice $F$. We first construct the lattice $F$ consisting of the elements $o, i$ and the elements $a_{p}, b_{p}$ for every $p \in P$, where $a_{p} \neq b_{p}$ for every $p \in P^{-}$and $a_{0}=b_{0}, a_{1}=b_{1}$. These elements are ordered and the lattice operations are formed as in Figure 2 .

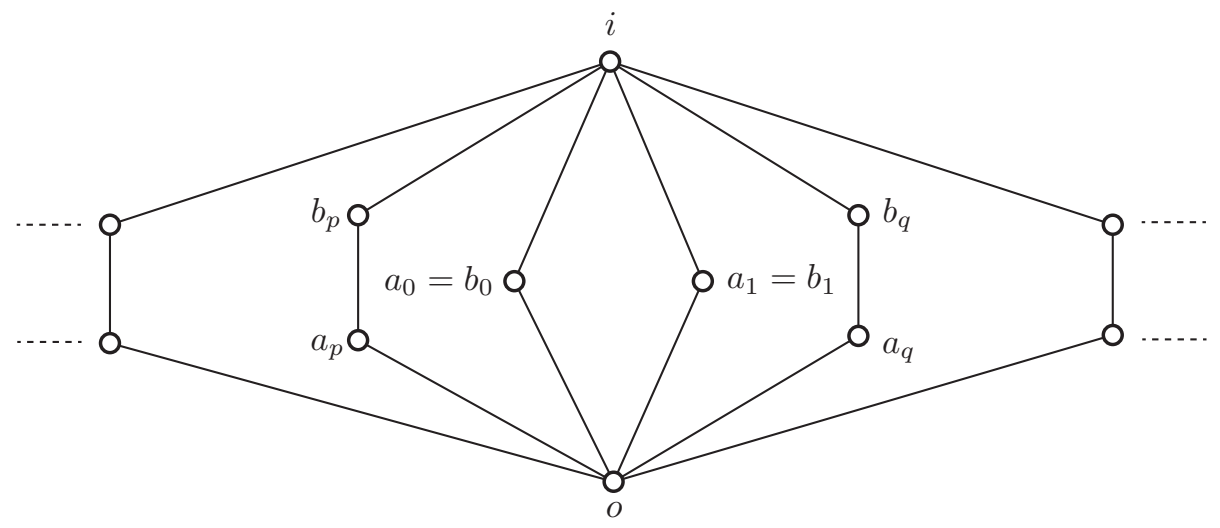

Figure 2. The lattice $F$

2.2. The lattice $K$. We are going to construct the lattice $K$ (of Theorem 2) as an extension of $F$. The principal congruence of $K$ representing $p \in P^{-}$will be $\operatorname{con}\left(a_{p}, b_{p}\right)$.

(a) We add the set

$$
\left\{c_{p, q}, d_{p, q}, e_{p, q}, f_{p, q}, g_{p, q}\right\}
$$

to the sublattice

$$
\left\{o, a_{p}, b_{p}, a_{q}, b_{q}, i\right\}
$$

of $F$ for $p<q \in P^{-}$to form the sublattice $S(p, q)$, as illustrated in Figure 3 .

(b) For $p \in P^{\mathrm{d}}$, let $C_{p}=\left\{o, a_{p}, b_{p}, i\right\}$, a four-element chain.

(c) We define the set

$$
K=\bigcup\left(S(p, q) \mid p<q \in P^{-}\right) \cup \bigcup\left(C_{p} \mid p \in P^{\mathrm{d}}\right) \cup\left\{a_{0}, a_{1}\right\} .
$$




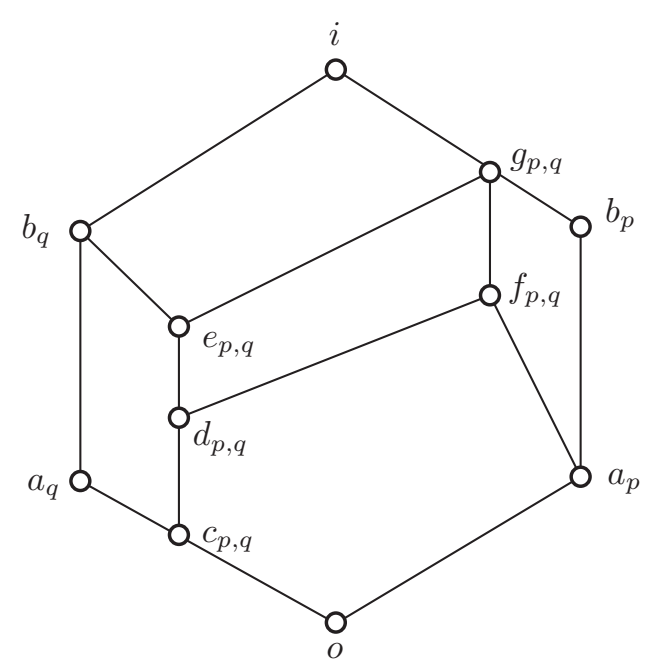

Figure 3 . The lattice $S=S(p, q)$

Now we are ready to define the lattice $K$.

We make the set $K$ into a lattice by defining the operations $\vee$ and $\wedge$ with the following nine rules.

(i) The operations $\vee$ and $\wedge$ are idempotent and commutative and $o$ is the zero and $i$ is the unit of $K$.

(ii) For $p \in P^{\mathrm{d}}$ and $x, y \in C_{p} \subseteq K$, we define $x \vee y, x \wedge y$ in $K$ as in the chain $C_{p}$. (So $C_{p}$ is a sublattice of $K$.)

(iii) For $p<q \in P^{-}$and $x, y \in S(p, q) \subseteq K$, we define $x \vee y, x \wedge y$ in $K$ as in the lattice $S(p, q)$. (So $S(p, q)$ is a sublattice of $K$.)

(iv) For $p \in P^{\mathrm{d}}, x \in C_{p}^{-}$, and $y \in K-C_{p}$, the elements $x$ and $y$ are complementary in $K$, that is, $x \vee y=i$ and $x \wedge y=o$.

(v) For $x=a_{0}$ and for $x=a_{1}$, the element $x$ is complementary to any element $y \neq x \in K^{-}$.

In the following four rules, let $p<q, p^{\prime}<q^{\prime} \in P^{-}, x \in S(p, q)^{-}$, and $y \in S\left(p^{\prime}, q^{\prime}\right)^{-}$. By rule (iii), we can assume that $\{p, q\} \neq\left\{p^{\prime}, q^{\prime}\right\}$.

(vi) If $\{p, q\} \cap\left\{p^{\prime}, q^{\prime}\right\}=\varnothing$, then the elements $x$ and $y$ are complementary in $K$.

(vii) If $q=p^{\prime}$, we form $x \vee y$ and $x \wedge y$ in $K$ in the lattice

$$
S_{\mathrm{C}}=S\left(p<q, q<q^{\prime}\right),
$$

illustrated in Figure 4

(viii) If $p=p^{\prime}$ and $q \neq q^{\prime}$, we form $x \vee y$ and $x \wedge y$ in $K$ in the lattice

$$
S_{\vee}=S\left(p<q, p<q^{\prime}\right),
$$

illustrated in Figure 5 


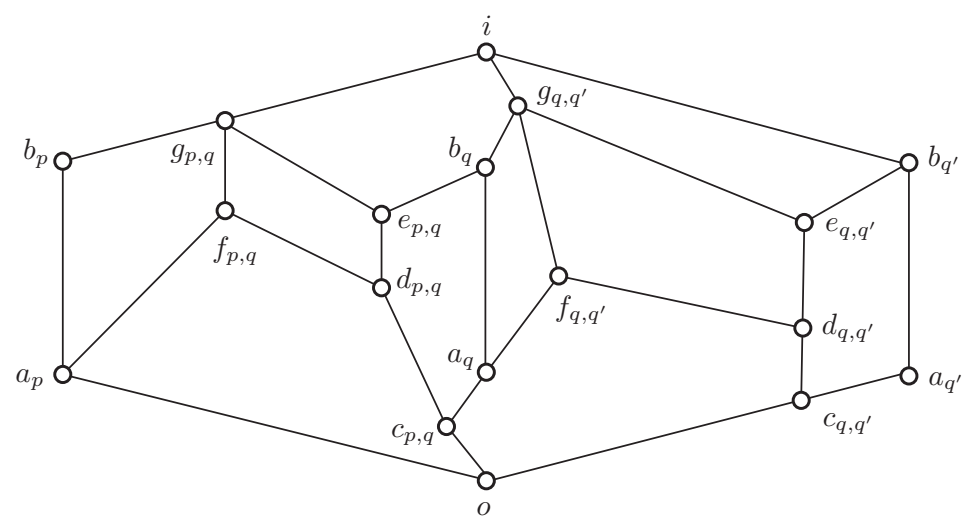

Figure 4 . The lattice $S_{\mathrm{C}}=S\left(p<q, q<q^{\prime}\right)$

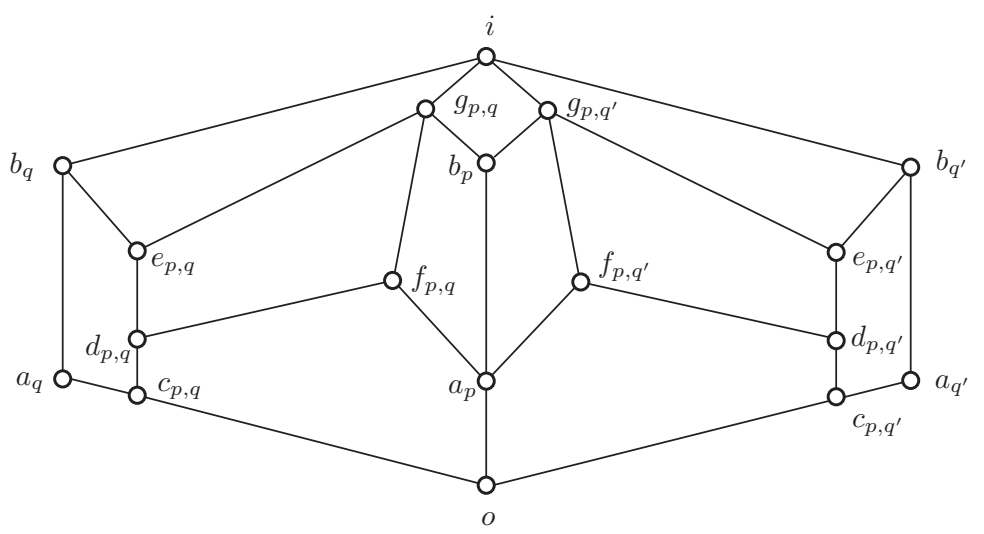

Figure 5. The lattice $S_{\vee}=S\left(p<q, p<q^{\prime}\right)$ with $q \neq q^{\prime}$

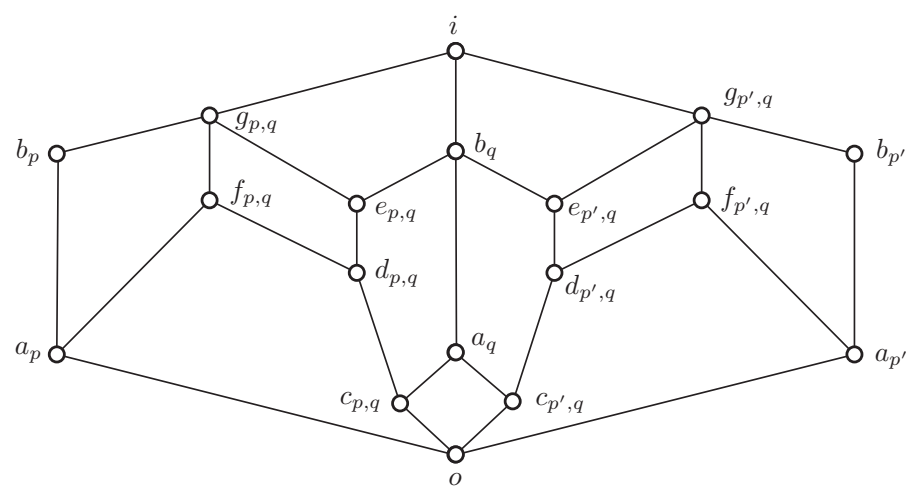

Figure 6 . The lattice $S_{\mathrm{H}}=S\left(p<q, p^{\prime}<q\right)$ with $p \neq p^{\prime}$ 
(ix) If $q=q^{\prime}$ and $p \neq p^{\prime}$, we form $x \vee y$ and $x \wedge y$ in $K$ in the lattice

$$
S_{\mathrm{H}}=S\left(p<q, p^{\prime}<q\right),
$$

illustrated in Figure 6 .

In the last three rules, $\mathrm{C}$ for chain, $\mathrm{V}$ for $\mathrm{V}$-shaped, $\mathrm{H}$ for Hat-shaped refer to the shape of the three element order $\{p, q\} \cup\left\{p^{\prime}, q^{\prime}\right\}$ in $P^{-}$.

Observe that Rules (vi)-(ix) exhaust all possibilities under the assumption $\{p, q\} \neq\left\{p^{\prime}, q^{\prime}\right\}$.

Note that

$$
\begin{aligned}
S & =S(p, q), \\
S_{\mathrm{C}} & =S\left(p<q, q<q^{\prime}\right), \\
S_{\bigvee} & =S\left(p<q, p<q^{\prime}\right), \\
S_{\mathrm{H}} & =S\left(p<q, p^{\prime}<q\right)
\end{aligned}
$$

are sublattices of $K$.

Informally, these rules state that to form $K$, we add elements to $F$ so that we get the sublattices listed in the previous paragraph.

Alternatively, we could have defined the ordering on $K$. Note that the ordering is larger than

$$
\bigcup\left(\leq_{S(p, q)} \mid p<q \in P^{-}\right) \cup \bigcup\left(\leq_{C_{p}} \mid p \in P^{\mathrm{d}}\right) .
$$

\section{The proof}

3.1. Preliminaries. It is easy, if somewhat tedious, to verify that $K$ is a lattice. Note that all our sublattice constructs are bounded planar orders, hence planar lattices. We have to describe the congruence structure of $K$.

Let $L$ be a lattice with 0 and 1 . A congruence block of $L$ is trivial if it is a singleton.

A $\{0,1\}$-isolating congruence $\boldsymbol{\alpha}$ of $L$ (an I-congruence, for short), is a congruence $\boldsymbol{\alpha}>\mathbf{0}$, such that $\{0\}$ and $\{1\}$ are (trivial) congruence blocks of $\boldsymbol{\alpha}$.

If $|P| \leq 2$, then we can construct $K$ as a one- or two-element chain. So for the proof, we assume that $|P|>2$, that is, $P^{-} \neq \varnothing$.

Lemma 3. For every $x \in K^{-}$, there is an $\{o, i\}$-sublattice $A$ of $K$ containing $x$ and isomorphic to $\mathrm{M}_{3}$.

Proof. Since $P^{-} \neq \varnothing$ by assumption, we can choose $p \in P^{-}$. If $x \in\left\{a_{0}, a_{1}\right\}$, then

$$
A=\left\{a_{p}, a_{0}, a_{1}, o, i\right\}
$$

is such a sublattice. If $x \notin\left\{a_{0}, a_{1}\right\}$, then

$$
A=\left\{x, a_{0}, a_{1}, o, i\right\}
$$

is such a sublattice. 
Lemma 4. Let us assume that $\boldsymbol{\alpha}$ is not an I-congruence of $K$. Then $\boldsymbol{\alpha}=\mathbf{1}$.

Proof. Indeed, if $\boldsymbol{\alpha}$ is not an I-congruence of $K$, then there is an $x \in K^{-}$such that $x \equiv o(\bmod \boldsymbol{\alpha})$ or $x \equiv o(\bmod \boldsymbol{\alpha})$. Using the sublattice $A$ provided by Lemma 3 , we conclude that $\boldsymbol{\alpha}=\mathbf{1}$, since $A$ is a simple $\{o, i\}$-sublattice.

3.2. The congruences of $S$. We start with the congruences of the lattice $S=S(p, q)$ with $p<q \in P^{-}$, see Figure 3 .

Lemma 5. The lattice $S=S(p, q)$ has two I-congruences:

$$
\operatorname{con}\left(a_{p}, b_{p}\right)<\operatorname{con}\left(a_{q}, b_{q}\right)
$$

see Figure 7
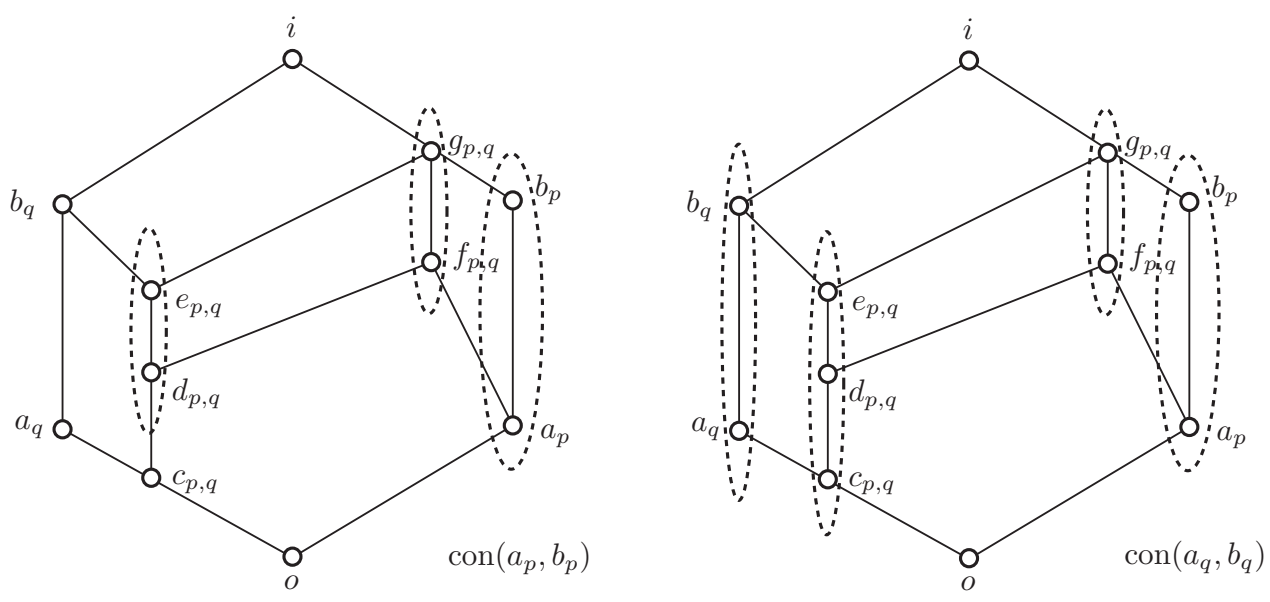

FiguRE 7. The I-congruences of $S=S(p, q)$

Proof. An easy computation. First, check that Figure 7 correctly describes the two join-irreducible I-congruences $\operatorname{con}\left(a_{p}, b_{p}\right)$ and $\operatorname{con}\left(a_{q}, b_{q}\right)$. Then, check all 12 prime intervals $[x, y]$ and show that $\operatorname{con}(x, y)$ is either not an I-congruence or equals $\operatorname{con}\left(a_{p}, b_{p}\right)$ or $\operatorname{con}\left(a_{q}, b_{q}\right)$. For instance, $\operatorname{con}\left(d_{p, q}, e_{p, q}\right)=\operatorname{con}\left(a_{p}, b_{p}\right)$ and $\left[b_{p}, g_{p, q}\right]$ is not an I-congruence because $c_{p, q} \equiv o\left(\bmod \operatorname{con}\left(b_{p}, g_{p, q}\right)\right)$. The other 10 cases are similar. Finally, note that the two join-irreducible I-congruences we found are comparable, so there are no join-reducible I-congruences.

Clearly, $S(p, q) / \operatorname{con}\left(a_{q}, b_{q}\right) \cong \mathrm{C}_{2} \times \mathrm{C}_{3}$.

3.3. The congruences of $K$. For $p \in P^{\mathrm{d}}$, let $\varepsilon_{p}$ denote the congruence $\operatorname{con}\left(a_{p}, b_{p}\right)$ on $K$.

Let $H \subseteq P^{\mathrm{d}}$. Let $\varepsilon_{H}$ denote the equivalence relation

$$
\varepsilon_{H}=\bigvee\left(\varepsilon_{p} \mid p \in H\right)
$$


on $K$.

Let $\boldsymbol{\beta}$ be an I-congruence of the lattice $K$. We associate with $\boldsymbol{\beta}$ a subset of the order $P^{-}$:

$$
\operatorname{Base}(\boldsymbol{\beta})=\left\{p \in P^{-} \mid a_{p} \equiv b_{p} \quad(\bmod \boldsymbol{\beta})\right\} .
$$

Lemma 6. Let $\boldsymbol{\beta}$ be an I-congruence of the lattice $K$. Then $\operatorname{Base}(\boldsymbol{\beta})$ is a down set of $P^{-}$.

Proof. Let $p<q \in P$ and let $q \in \operatorname{Base}(\boldsymbol{\beta})$. Then $a_{q} \equiv b_{q}(\bmod \boldsymbol{\beta})$. By Lemma 5 (see also Figure 7), $a_{p} \equiv b_{p}(\bmod \boldsymbol{\beta})$, so $p \in \operatorname{Base}(\boldsymbol{\beta})$, verifying that $\operatorname{Base}(\boldsymbol{\beta})$ is a down set.

Let $H$ be a down set of $P^{-}$. We define the binary relation:

$$
\boldsymbol{\beta}_{H}=\varepsilon_{H} \cup \bigcup\left(\operatorname{con}_{S(p, q)}\left(a_{q}, b_{q}\right) \mid q \in H\right) \cup \bigcup\left(\operatorname{con}_{S(p, q)}\left(a_{p}, b_{p}\right) \mid p \in H\right) \text {. }
$$

Lemma 7. $\boldsymbol{\beta}_{H}$ is an I-congruence on $K$.

Note that $\boldsymbol{\beta}_{\varnothing}=\mathbf{0}$.

Proof. $\boldsymbol{\beta}_{H}$ is reflexive and symmetric. It clearly leaves $o$ and $i$ isolated.

It is easy to verify that $\boldsymbol{\beta}_{H}$ classes are pairwise disjoint two- and threeelement chains, so $\boldsymbol{\beta}_{H}$ is transitive and hence an equivalence relation.

We verify the Substitution Properties. By Lemma I.3.11 of [3], it is sufficient to verify that if $x<y \in K$, and $x \equiv y\left(\bmod \boldsymbol{\beta}_{H}\right)$, then $x \vee z \equiv y \vee z\left(\bmod \boldsymbol{\beta}_{H}\right)$ and $x \wedge z \equiv y \wedge z\left(\bmod \boldsymbol{\beta}_{H}\right)$ for $z \in K$.

So let $x<y \in K^{-}$and $x \equiv y\left(\bmod \boldsymbol{\beta}_{H}\right)$. Then $x<y \in S(p, q)^{-}$, for some $p<q \in P^{-}$, and

$$
x \equiv y \quad\left(\bmod \operatorname{con}_{S(p, q)}\left(a_{q}, b_{q}\right)\right)
$$

with $q \in H$, or

$$
x \equiv y \quad\left(\bmod \operatorname{con}_{S(p, q)}\left(a_{p}, b_{p}\right)\right)
$$

with $p \in H$.

Let $z \in S\left(p^{\prime}, q^{\prime}\right)^{-}$with $p^{\prime}<q^{\prime} \in P^{-}$.

If $\{p, q\}=\left\{p^{\prime}, q^{\prime}\right\}$, the Substitution Properties for $\boldsymbol{\beta}_{H}$ in $K$ follow from the Substitution Properties for con $\left(a_{p}, b_{p}\right)$ in $S(p, q)$.

If $\{p, q\} \cap\left\{p^{\prime}, q^{\prime}\right\}=\varnothing$, then by Rule (vi), the elements $x, z$, and $y, z$ are complementary, so the Substitution Properties are trivial.

Otherwise, $\{p, q\} \cup\left\{p^{\prime}, q^{\prime}\right\}$ has three elements. So we have three cases to consider.

Case C: $p<p^{\prime}=q<q^{\prime}$ (or symmetrically, $p^{\prime}<q^{\prime}=p<q$ ).

Case V: $p=p^{\prime}<q, p=p^{\prime}<q^{\prime}, q \neq q^{\prime}$.

Case $\mathrm{H}: p<q=q^{\prime}, p^{\prime}<q=q^{\prime}, p \neq p^{\prime}$.

To verify Case $C$, utilize Figure 4 . Since

$$
x \leq y \in S(p, q)^{-} \subseteq S\left(p<q, q<q^{\prime}\right)^{-}
$$

and

$$
z \in S\left(q, q^{\prime}\right)^{-} \subseteq S\left(p<q, q<q^{\prime}\right)^{-},
$$


there is only way $\left(\mathrm{SP}_{\vee}\right)$ can fail: $x \vee z<y \vee z$.

We can assume that $z \notin S(p<q)$, so $x \vee z, y \vee z \notin S(p<q)$. If $q \in H$, then there is only one case to check for the I-congruence $\boldsymbol{\beta}_{H}$ :

$$
(x \vee z, y \vee z)=\left(f_{q, q^{\prime}}, g_{q, q^{\prime}}\right) \in \operatorname{con}\left(a_{q}, b_{q}\right) \subseteq \boldsymbol{\beta}_{H} .
$$

If $q \notin H$, then $p \in H$ and $x \vee z<y \vee z$ is impossible. This shows that $\boldsymbol{\beta}_{H}$ satisfies $\left(\mathrm{SP}_{\vee}\right)$. A similar, in fact easier, argument yields $\left(\mathrm{SP}_{\wedge}\right)$.

We leave Case $\mathrm{V}$ and Case $\mathrm{H}$ to the reader.

Now the following statement is clear.

Lemma 8. The correspondence

$$
\varphi: \boldsymbol{\beta} \rightarrow \operatorname{Base}(\boldsymbol{\beta})
$$

is an order preserving bijection between the order of I-congruences of $K$ and the order of down sets of $P^{-}$. We extend $\varphi$ by $\mathbf{0} \rightarrow\{0\}$ and $\mathbf{1} \rightarrow P$. Then $\varphi$ is an isomorphism between Con $K$ and Down $^{-} P$, the order of nonempty down sets of $P$.

Lemma 9. $\varphi$ and $\varphi^{-1}$ both preserve the property of being principal.

Proof. Indeed, if the I-congruence $\boldsymbol{\beta}$ of $K$ is principal, $\boldsymbol{\beta}=\operatorname{con}(x, y)$ for some $x<y \in K$, then we must have $x, y \in S(p, q)$ for some $p<q \in P^{-}$(otherwise, $\boldsymbol{\beta}$ would not be an I-congruence). But in $S(p, q)$ (see Figure 7), the principal congruences are $\operatorname{con}\left(a_{p}, b_{p}\right)$ and $\operatorname{con}\left(a_{q}, b_{q}\right)$. By Lemma 8 , we obtain that $\operatorname{Base}(\boldsymbol{\beta})=\downarrow p$ or $\operatorname{Base}(\boldsymbol{\beta})=\downarrow q$.

Conversely, if $\operatorname{Base}(\boldsymbol{\beta})=\downarrow p$, then $\boldsymbol{\beta}=\operatorname{con}\left(a_{p}, b_{p}\right)$.

Now Theorem 2 easily follows. Indeed, by Lemma 8 , $\varphi$ is an isomorphism between Con $K$ and Down ${ }^{-} P$. Under this isomorphism, by Lemma 9 , principal congruences correspond to principal down sets, so Princ $K \cong P$, as claimed.

Note added in proof. R. W. Quackenbush has just sent me a manuscript of his with P. P. Pálfy, The representation of principal congruences, accepted for publication in 1993. The final version was not submitted.

Anybody interested in the universal algebraic problems of Section 1.4.5 should read this manuscript.

\section{REFERENCES}

[1] Birkhoff, G.: Lattice Theory. Amer. Math. Soc. Colloq. Publ. vol. 25, rev. ed. Amer. Math. Soc., New York (1948)

[2] Grätzer, G.: The Congruences of a Finite Lattice, A Proof-by-Picture Approach. Birkhäuser Boston (2006)

[3] Grätzer, G.: Lattice Theory: Foundation. Birkhäuser Verlag, Basel (2011)

[4] Grätzer, G., Lakser, H., and Schmidt, E. T.: Congruence lattices of finite semimodular lattices, Canad. Math. Bull. 41, 290-297 (1998)

[5] Grätzer, G. and Schmidt, E. T.: On inaccessible and minimal congruence relations. I. Acta Sci. Math. (Szeged) 21, 337-342 (1960) 
[6] _ : On congruence lattices of lattices. Acta Math. Acad. Sci. Hungar. 13, $179-185(1962)$

[7] _ : A lattice construction and congruence-preserving extensions. Acta Math. Hungar. 66, 275-288 (1995)

[8] _ : Congruence-preserving extensions of finite lattices to semimodular lattices. Houston J. Math. 27, 1-9 (2001)

[9] __ : Regular congruence-preserving extensions of lattices. Algebra Universalis 46, $119-130(2001)$

[10] _ : On the Independence Theorem of related structures for modular (arguesian) lattices. Studia Sci. Math. Hungar. 40, 1-12 (2003)

[11] Grätzer, G. and Wehrung, F.: Proper congruence-preserving extensions of lattices. Acta Math. Hungar. 85, 175-185 (1999)

[12] Grätzer G. and Wehrung, F., editors: Lattice Theory: Empire. Special Topics and Applications. Birkhäuser, Basel, forthcoming.

[13] Ore, O.: Theory of equivalence relations. Duke Math. J. 9, 573-627 (1942)

[14] Schmidt, E. T.: Über die Kongruenzverbänder der Verbände. Publ. Math. Debrecen 9, 243-256 (1962)

[15] Wehrung, F.: A solution to Dilworth's congruence lattice problem. Adv. Math. 216, $610-625(2007)$

G. GRÄTZER

Department of Mathematics, University of Manitoba, Winnipeg, MB R3T 2N2, Canada e-mail, G. Grätzer: gratzer@me.com

URL, G. Grätzer: http://server.maths.umanitoba.ca/homepages/gratzer/ 